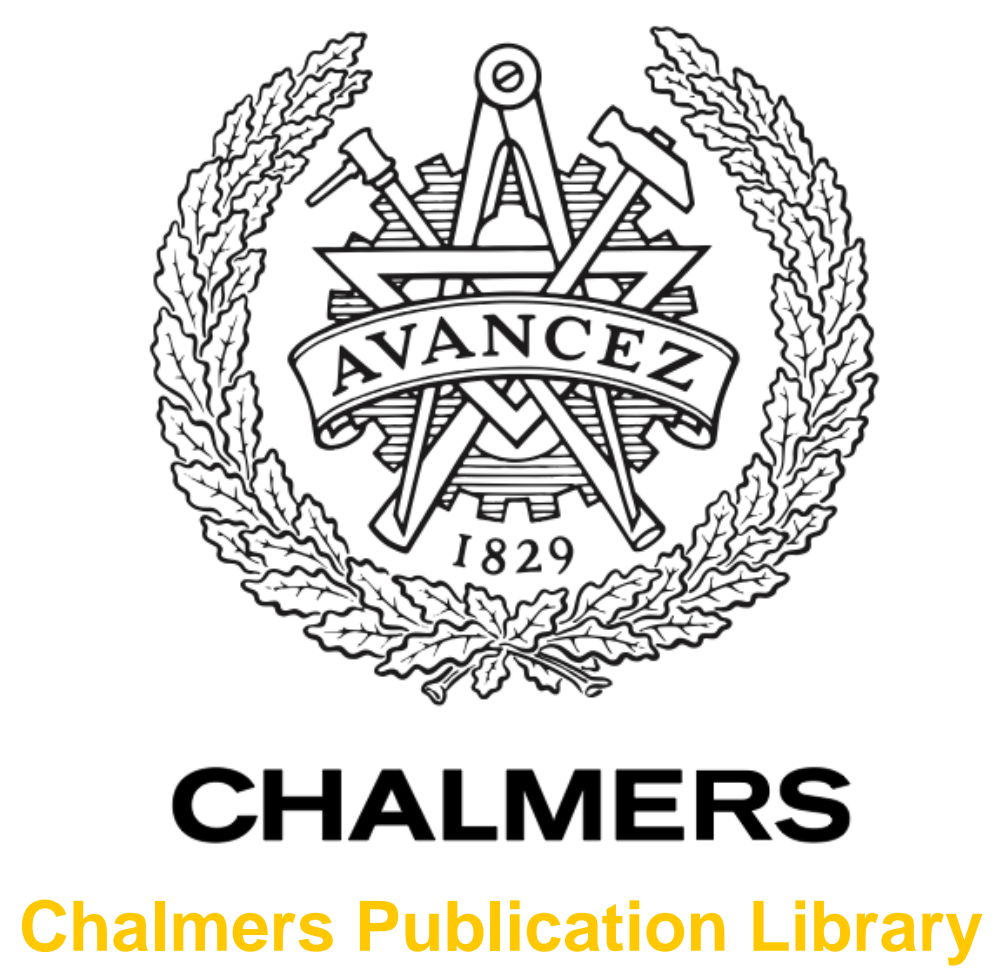

\title{
Formation and switching of defect dipoles in acceptor-doped lead titanate: A kinetic model based on first-principles calculations
}

This document has been downloaded from Chalmers Publication Library (CPL). It is the author's version of a work that was accepted for publication in:

Physical Review B (ISSN: 1098-0121)

Citation for the published paper:

Erhart, P. ; Träskelin, P. ; Albe, K. (2013) "Formation and switching of defect dipoles in acceptor-doped lead titanate: A kinetic model based on first-principles calculations". Physical Review B, vol. 88(2), pp. 024107.

http://dx.doi.org/10.1103/PhysRevB.88.024107

Downloaded from: http://publications.lib.chalmers.se/publication/181510

Notice: Changes introduced as a result of publishing processes such as copy-editing and formatting may not be reflected in this document. For a definitive version of this work, please refer to the published source. Please note that access to the published version might require a subscription. 


\title{
Formation and switching of defect dipoles in acceptor-doped lead titanate: A kinetic model based on first-principles calculations
}

\author{
Paul Erhart, ${ }^{1, *}$ Petra Träskelin, ${ }^{2}$ and Karsten Albe ${ }^{3}$ \\ ${ }^{1}$ Chalmers University of Technology, Department of Applied Physics, SE-412 96 Gothenburg, Sweden \\ ${ }^{2}$ University of Gothenburg, Department of Physics, SE-412 96 Gothenburg, Sweden \\ ${ }^{3}$ Technische Universität Darmstadt, Institut für Materialwissenschaft, D-64287 Darmstadt, Germany
}

(Received 20 February 2013; published 11 July 2013)

\begin{abstract}
The formation and field-induced switching of defect dipoles in acceptor doped lead titanate is described by a kinetic model representing an extension of the well established Arlt-Neumann model [Ferroelectrics 76, 303 (1987)]. Energy barriers for defect association and reorientation of oxygen vacancy-dopant ( $\mathrm{Cu}$ and $\mathrm{Fe}$ ) complexes are obtained from first-principles calculations and serve as input for kinetic coefficients of the rate equation model. The numerical solution of the model describes the time evolution of the oxygen vacancy distribution at different temperatures and dopant concentrations in the presence or absence of an alternating external field. We predict the characteristic time scale for the alignment of all defect dipoles with the spontaneous polarization of the surrounding matrix. In this state the defect dipoles act as obstacles for domain wall motion and contribute to the experimentally observed aging. Under cycling conditions the fully aligned configuration is perturbed and a dynamic equilibrium is established with defect dipoles in parallel and antiparallel orientation relative to the spontaneous polarization. This process can be related to the deaging behavior of piezoelectric ceramics.
\end{abstract}

DOI: 10.1103/PhysRevB.88.024107

PACS number(s): 61.72.jd, 71.15.Mb, 77.80.Fm, 77.84.Cg

\section{INTRODUCTION}

Aging phenomena, namely the gradual change of physical properties with time, are observed in almost all ferroelectrics. ${ }^{1-13}$ In acceptor doped barium titanate $\left(\mathrm{BaTiO}_{3}\right)$ and lead zirconate titanate (PZT) ceramics aging usually leads to an increasing shift of the hysteresis along the axis of the electrical field giving rise to an internal bias field. ${ }^{14}$ In the past, several plausible models have been developed to interpret the occurrence of bias fields and aging phenomena in ferroelectrics in terms of domain splitting, ${ }^{2}$ space-charge formation, ${ }^{4}$ electronic charge trapping, ${ }^{7,8}$ ionic drift, ${ }^{11}$ and reorientation of defect dipoles. ${ }^{5,15-17}$

In acceptor ("hard") doped ferroelectrics transition metals usually substitute the $B$-site (occupied by Ti or Zr in PZT) and tend to bind strongly to oxygen vacancies. These acceptor center-oxygen vacancy associates form electric and elastic defect dipoles such as charged $\left(\mathrm{Fe}_{\mathrm{Zr}, \mathrm{Ti}}^{\prime}{ }^{-} V_{\mathrm{O}}^{\bullet \bullet}\right)^{\bullet}$ or $\left(\mathrm{Cu}_{\mathrm{Zr}, \mathrm{Ti}^{-}}^{\prime \prime}\right.$ $\left.V_{\mathrm{O}}^{\bullet \bullet}\right)^{\times},{ }^{18,19}$ which contribute to the overall polarization in a ferroelectric compound ${ }^{7,18-24}$ and can be aligned either parallel, antiparallel, or perpendicular to the polarization of the surrounding material as shown schematically in Fig. 1.

In the paraelectric state, defect dipoles of different orientation are energetically equivalent, whereas they have a preferred orientation in a polar matrix. Arlt and Neumann ${ }^{5,25}$ have attributed the occurrence of internal bias fields to the switching of defect dipoles and described the transient orientation of dipoles by a kinetic model. As quantitative data on the energy landscape for these defect dipoles was unavailable at the time they relied on a simple electrostatic estimate of the energy difference. ${ }^{26}$ The energetic asymmetry between the parallel and antiparallel dipoles obtained in this fashion for $\mathrm{BaTiO}_{3}$ was about $30 \mathrm{meV}$ and thus much smaller than the energy differences calculated more recently by first-principles methods for $\mathrm{PbTiO}_{3}$, ${ }^{19-21}$ which revealed that the energetic asymmetry is actually as large as the barriers for oxygen migration. Only recently, Marton and Elsässer ${ }^{24}$ showed that in Fe-doped lead titanate the barrier for reorientation sensitively depends on the position of the migrating oxygen vacancy with respect to the iron atom and the surrounding spontaneous ferroelectric polarization. During fast field cycling, the defect dipoles are expected not to change orientation, because the characteristic rate for oxygen jumps around the acceptor center should be lower than the frequency of the domain switching process. Experimentally, Zhang et al. ${ }^{10}$ followed the dynamics of $\left(\mathrm{Mn}_{\mathrm{Ti}}-V_{\mathrm{O}}\right)^{\times}$dipoles in barium titanate by electron paramagnetic resonance studies and found support for the so-called "defect symmetry principle," which assumes that nonswitching defect dipoles impose a restoring force for reversible domain switching. ${ }^{15}$ Jakes et al. could show that in $\mathrm{Fe}^{3+}$ doped PZT defect dipoles are not preferentially located at domain walls but within the domains. ${ }^{27}$ Morozov et al. studied aging-deaging process in hard PZT ceramics using the harmonic analysis of polarization response under switching conditions and concluded that two or more mechanisms are responsible for domain stabilization. ${ }^{11}$ Activation energies of about $0.6 \mathrm{eV}$ were attributed to short-range charge hopping, which could be due to local reorientation of defect dipoles. Since the switching dynamics of defect dipoles depends on the electric and thermal energy provided to change polarization direction, the contribution of dipole reorientation can only be reliably assessed if realistic numbers for the migration and association energies are available, which allow us to quantitatively model the switching dynamics of defect dipoles in a comprehensive way.

The objective of the present work is to develop a kinetic model that captures the formation of defect dipoles as well as their reorientation both in the absence and presence of electric fields. ${ }^{28} \mathrm{Cu}$ and $\mathrm{Fe}$-doped lead titanate are considered as representative examples, and the energy landscape for oxygen vacancy migration in these materials is obtained 

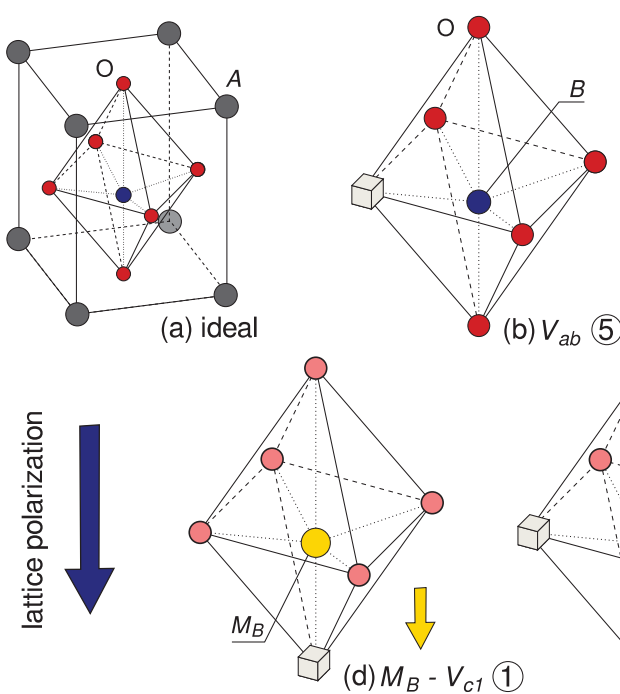

(b) $V_{a b}$ (5)

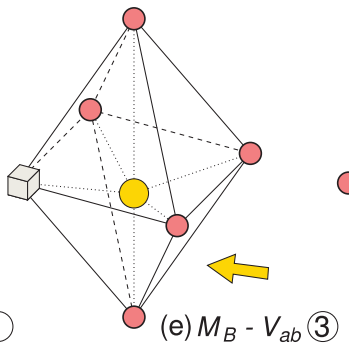

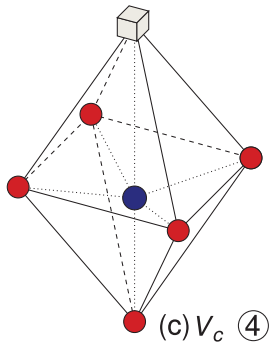

(c) $V_{c}$ (4)
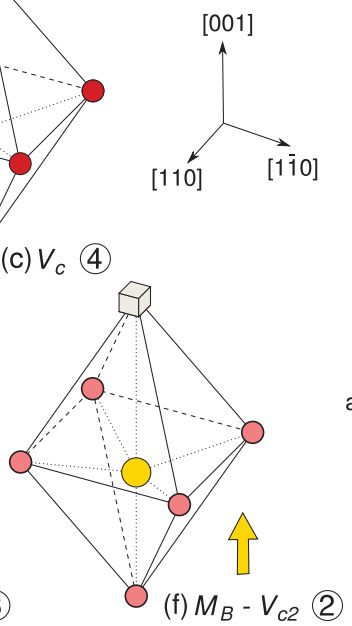
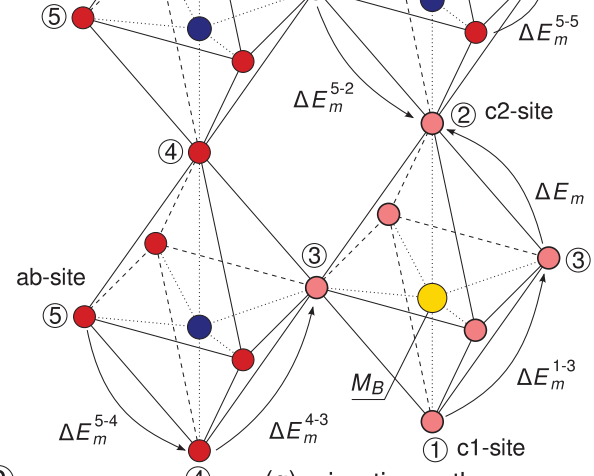

(g) migration paths

FIG. 1. (Color online) (a) Unit cell of the ideal tetragonal perovskite $\left(A B \mathrm{O}_{3}\right)$ lattice. (b),(c) Free oxygen vacancies. (d)-(f) Complexed oxygen vacancies on nearest-neighbor sites of $B$-site impurities. The dark blue arrow on the left indicates the direction of the lattice polarization whereas the smaller yellow arrows represent the orientation of the defect dipoles. (g) Schematic representation of the possible paths for oxygen vacancy migration. Some migration barriers, $\Delta E_{m}^{i-j}$, are exemplarily indicated. In (b)-(g) only the $B$ and $\mathrm{O}$ sites are shown. Oxygen vacancies jump along the vertices of the $B_{6}$ octahedron. Blue and yellow circles represent native and impurity atoms on $B$ sites, respectively. The dark and light red circles indicate oxygen atoms in the first nearest neighbor shell of native and impurity atoms, respectively.

using first-principles calculations. We consider both free oxygen vacancies and oxygen vacancies associated with $\mathrm{Fe}$ or $\mathrm{Cu}$. Starting from a statistical distribution the majority of oxygen vacancies is initially unbound. Over time vacancies are captured by impurity atoms and subsequently converted into the lowest energy configuration, which corresponds to a defect dipole that is aligned parallel to the macroscopic polarization $\left(M_{B}-V_{c 1}\right.$ in Fig. 1). While the exact time scales for these processes are dependent on dopant type, concentration, and temperature, our results demonstrate that slightly above room temperature the ground state is reached within seconds and thus the pristine material can already be considered as "aged." In the presence of an oscillating external field our model predicts that gradual reorientation of defect dipoles leads to a dynamic equilibrium, in which the parallel and antiparallel configurations occur with equal probability. This is in accord with the experimental observation of deaging by the application of AC fields. ${ }^{29,30}$

This paper is organized as follows. First, we describe the kinetic model and discuss its features. This is followed in Sec. III by a description of the first-principles calculations that were carried out to determine the model parameters. In Sec. IV we apply the kinetic model to study vacancy redistribution as a function of temperature and impurity concentration both in the absence and presence of an oscillating external electric field. The implications of the present findings for aging and fatigue are discussed in Sec. V and conclusions are summarized in Sec. VI.

\section{KINETIC MODEL}

In this section we formulate a kinetic model that describes the redistribution of oxygen vacancies between different types of sites as a function of time. It captures the temperature, impurity concentration, and frequency dependence of this process within a mean-field approximation. Figure 1 provides an overview of the different types of oxygen vacancies that are taken into account by this model.

In general, the temporal variation of the concentration of vacancies of type $i$ can be described by a rate equation

$$
\frac{\partial c_{i}}{\partial t}=-\sum_{j} \Phi_{i j} \mathrm{~K}_{i j} c_{i}+\sum_{j} \Phi_{j i} \mathrm{~K}_{j i} c_{j},
$$

where the first term on the right-hand side accounts for the "loss" of vacancies of type $i$ while the second term describes the "gain" due to vacancy jumps from sites of type $j$ to sites of type $i$. At typical device operation temperatures near $300 \mathrm{~K}$ the creation or annihilation of vacancies at surfaces or interfaces is negligible and the total concentration of vacancies can be assumed as constant,

$$
\sum_{i} c_{i}=c_{\mathrm{tot}} .
$$

Following classical transition state theory and invoking a harmonic approximation the rate, at which vacancies of type $i$ jump onto sites of type $j$, can be expressed as

$$
\mathrm{K}_{i j}=v_{0}^{i} \exp \left(-\frac{\Delta E_{m}^{i-j}}{k_{B} T}\right),
$$

where $v_{0}^{i}$ is the attempt frequency and $\Delta E_{m}^{i-j}$ is the migration energy encountered by a vacancy jumping from a site of type $i$ to a site of type $j$.

The probability $\Phi_{i j}$ for a vacancy to jump from a site of type $i$ to a site of type $j$ is given by the fraction of sites of type $j$ in the first nearest neighbor shell of sites of type $i$. We employ a simple mapping to index different defect configurations, $M_{B}-V_{c 1} \rightarrow(1), M_{B}-V_{c 2} \rightarrow$ (2), $M_{B}-V_{a b} \rightarrow$ (3), $V_{c} \rightarrow$ (4), and 
$V_{a b} \rightarrow(5)$ [see Fig. 1(g) for examples]. Taking into account the geometry of the lattice (see Fig. 1) one then obtains the following probability matrix

$$
\boldsymbol{\Phi}=\frac{1}{8}\left(\begin{array}{ccccc}
0 & 0 & 4 & 0 & 4 \\
0 & 0 & 4 & 0 & 4 \\
1 & 1 & 2 & 2 & 2 \\
0 & 0 & 8 \alpha & 0 & 8(1-\alpha) \\
4 \alpha & 4 \alpha & 8 \alpha & 8(1-\alpha) & 8(1-\alpha)
\end{array}\right)
$$

where $\alpha=6 f_{M}$. Here $f_{M}$ is the fraction of $B$ sites which have been replaced by impurity atoms. The recurrence of the factor eight in Eq. (4) results from the number of oxygen sites in the second neighbor shell of any given oxygen site, while the factor six stems from the number of oxygen sites in the first neighbor shell of a $B$ site. Introducing $\mathrm{W}_{i j}=\Phi_{i j} \mathrm{~K}_{i j}$ and $\mathrm{V}_{i j}=\delta_{i j} \sum_{k} \mathrm{~W}_{i k}$, Eq. (1) can be rewritten in a convenient matrix form

$$
\dot{\boldsymbol{c}}=\left(\mathbf{W}^{\mathrm{T}}-\mathbf{V}\right) \boldsymbol{c}
$$

which in this work has been solved numerically ${ }^{31}$ using an adaptive time step algorithm for stiff differential equations. ${ }^{32}$

It should be noted that the model does not take into account the possibility of two or more oxygen vacancies associating with the same impurity atom. Both experiments and calculations indicate, however, that this is unlikely to occur for the material and dopants considered in the present work. Similarly, the possibility that two or more impurity atoms form an aggregate can be ruled out based on experimental evidence. $^{18}$

In the present form the model does not include any explicit constraints to allow for the number of oxygen vacancies to be larger than the number of impurity atoms or vice versa. This situation can, however, be implemented rather easily by solving the kinetic model in steps. For instance, consider a case in which the vacancy concentration is $\left[V_{\mathrm{O}}\right]=0.01$ and the dopant/impurity concentration is $[M]=0.005$. The sum of the relative concentrations of complexed vacancies $[M] /\left[V_{\mathrm{O}}\right]$ can, therefore, not exceed $c_{\max }=0.5>c_{1}+c_{2}+c_{3}$. Starting from some initial distribution, one solves the kinetic model until $c_{1}+c_{2}+c_{3}$ equals $c_{\max }$. At this point all impurities are complexed with vacancies, and one can "remove" the free vacancy concentrations $c_{4}$ and $c_{5}$ from the model. This is achieved by reducing the $5 \times 5$ matrices in Eqs. (1)(4) and Eq. (5) to $3 \times 3$ matrices, only keeping elements $(i, j) \in\{1,2,3\}$. The opposite scenario, in which the number of impurity/dopant atoms exceeds the number of free vacancies, can be implemented in a similar fashion. For the sake of clarity and because the key conclusions of this work are unaffected, we do not consider any of these cases in the remainder of this paper.

Applying the model to a specific material requires knowledge of the attempt frequencies $v_{0}^{i}$ as well as the migration energies $\Delta E_{m}^{i-j}$ that enter Eq. (3). The latter are the most important quantities since they enter the exponential temperature dependence. In the present work we have obtained these numbers using first-principles calculations as described in the following section. Within classical transition state theory the attempt frequency for path $i$ is given by the ratio ${ }^{33,34}$

$$
v_{0}^{i}=\frac{1}{2 \pi} \frac{\prod_{k=1}^{N} \omega_{k}}{\prod_{k=1}^{N-1} \omega_{k}^{\dagger}},
$$

where $\omega_{k}$ and $\omega_{k}^{\dagger}$ are the normal mode frequencies for the ground and transition state, respectively. The attempt frequency $v_{0}^{i}$ for path $i$ can thus be computed via a vibrational analysis of the system (see e.g., Ref. 35). In the present case, all migration events of interest involve the motion of oxygen atoms between nearest neighbor sites; one can thus expect that variations in $v_{0}^{i}$ between different paths are small. We therefore abstained from an explicit calculation of $v_{0}^{i}$ and instead invoked a simple approximation. It is reasonable to assume that most vibrational modes are similar for ground and transition state, and thus the ratio Eq. (6) will be dominated by the frequency that is missing from the transition state term and that corresponds to the unstable mode at the saddle point, i.e., $\nu_{0} \approx \omega_{t r} / 2 \pi$. For simplicity we approximated this frequency with the lowest optical mode at $\Gamma, v_{0} \approx 2 \mathrm{THz},{ }^{36}$ which represents a characteristic frequency for atomic vibrations. According to Eq. (3) $v_{0}^{i}$ has a linear effect on $v_{i}$ and thus a change in the attempt frequency merely corresponds to a rescaling of the rates.

\section{FIRST-PRINCIPLES CALCULATIONS}

\section{A. Computational parameters}

Binding energies and barriers for oxygen vacancy migration in pure as well as $\mathrm{Cu}$ and Fe-doped lead titanate were calculated within density functional theory (DFT) using the Vienna $a b$ initio simulation package. ${ }^{37}$ The potentials due to the ions and the core electrons were represented by the projector-augmented wave method. ${ }^{38}$ The $5 d$ electrons of $\mathrm{Pb}$, the $3 s$ and $3 p$ electrons of Ti, as well as the $3 p$ electrons of $\mathrm{Fe}$ and $\mathrm{Cu}$ were treated as part of the valence. The exchangecorrelation potential was represented using the local spin density approximation. ${ }^{39}$ For migration barrier calculations supercells containing $3 \times 3 \times 3$ unit cells equivalent to 135 atoms were employed. The distance dependence of the binding between oxygen vacancies and $\mathrm{Cu}$ impurities was determined using supercells with $4 \times 4 \times 4$ unit cells equivalent to 320 atoms. In all cases the Brillouin zone was sampled using a $2 \times 2 \times 2$ Monkhorst-Pack mesh. Similar computational parameters were successfully used in previous studies of $\mathrm{Cu}$ and Fe-doped lead titanate. ${ }^{18,19,23}$

All computations were performed at the theoretical lattice constant of $a_{0}=3.866 \AA$ and the theoretical value for the axial ratio of $c / a=1.05$, both of which are in reasonable agreement with experiment $\left(a_{0}=3.905 \AA, c / a=1.064\right.$ at room temperature, Refs. 40 and 41 ). The calculated band gap of $1.47 \mathrm{eV}$ is considerably smaller than the experimental value, but consistent with the well known band gap error of DFT. As argued in Ref. 42 the band gap error is, however, expected to have a minor effect in the context of migration barrier calculations. Migration paths and barriers were determined using the climbing image nudged elastic band method ${ }^{43,44}$ and configurations were optimized until the maximum force was less than $20 \mathrm{meV} / \AA$. For charged defects a homogeneous background charge was added. We tested the convergence of 
the calculated binding energies and migration barriers both with respect to $k$-point sampling and system size and found variations of less than $0.05 \mathrm{eV} .{ }^{45}$

\section{B. Binding energies}

The binding between $\mathrm{Fe}$ and $\mathrm{Cu}$ impurities and oxygen vacancies has been studied previously using first-principles calculations. ${ }^{18,19,23}$ In the context of the present work it is, however, instructive to briefly revisit this aspect. One of the simplifications of the kinetic model described in Sec. II is the restriction to first nearest neighbor impurity-vacancy pairs. Computational resources restricted the system sizes accessible in earlier studies and prevented an extensive study of this aspect. To close this gap, we have constructed all symmetry inequivalent impurity-vacancy pairs that are accessible in 135 atom $(3 \times 3 \times 3$ unit cells $)$ and 320 atom $(4 \times 4 \times 4)$ supercells, which yields 21 and 48 distinct configurations, respectively. The results of the calculations, which were carried out for the case of neutral $\mathrm{Cu}_{\mathrm{Ti}}-V_{\mathrm{O}}$ complexes, are shown in Fig. 2.

As in the earlier studies ${ }^{18,19,23}$ the $\mathrm{Cu}_{\mathrm{Ti}}-V_{c 1}$ configuration is the most strongly bound complex, followed by the other two first nearest neighbor configurations. Among the 24 second nearest neighbor pairs (including symmetry equivalent configurations) 16 configurations are unstable and relax into first nearest neighbor complexes. ${ }^{46}$ The remaining eight configurations ${ }^{47}$ are stable and their interaction energy is within $0.05 \mathrm{eV}$ of the noninteracting limit. The latter is indicated by the horizontal gray dashed line and corresponds to the saturation of the interaction energy clearly evident for the complexes with the largest separation in the case of the 320 atom cell calculations.

The interaction strength thus decreases very quickly with distance as already the stable second neighbor pairs at a distance of about $4 \AA$ are energetically very close to the noninteracting limit. A small exception are the two (out of 30) third nearest neighbor configurations marked by the

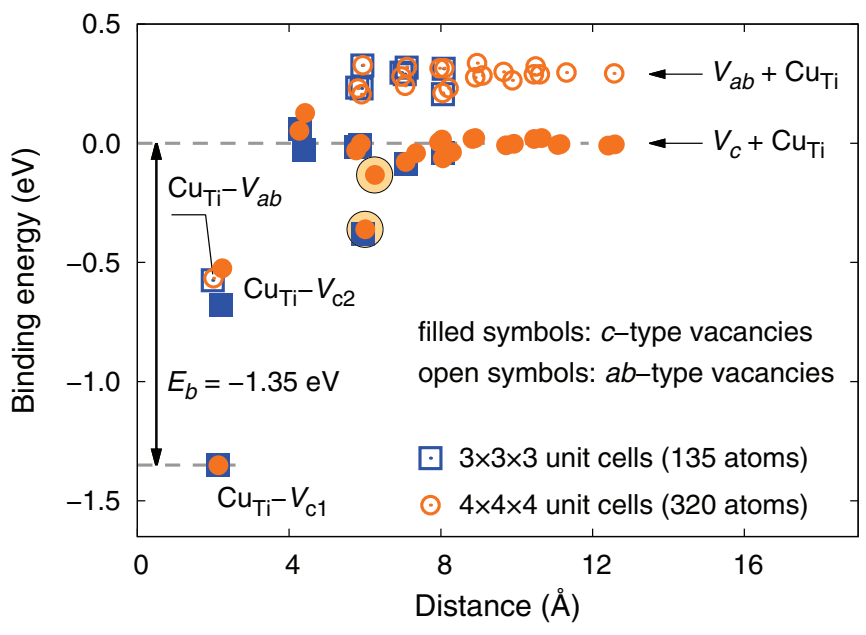

FIG. 2. (Color online) Binding energy of $\mathrm{Cu}_{\mathrm{Ti}}-V_{\mathrm{O}}$ complexes as a function of the distance between the $\mathrm{Cu}$ atom at the vacancy center. The gray horizontal line indicates the noninteracting limit. The two configurations marked by shaded circles correspond to third nearest neighbor impurity-vacancy complexes that are aligned along [001]. shaded circles in Fig. 2 that exhibit binding energies of -0.36 and $-0.13 \mathrm{eV}$. In these structures, which have already been investigated in Ref. 19, vacancy and impurity atom are aligned along [001].

These results thus support the construction of the model. They also provide a revised value ${ }^{48}$ for the binding energy of $\mathrm{Cu}_{\mathrm{Ti}}-V_{\mathrm{O}}$ of $-1.35 \mathrm{eV}$, which will be employed in the construction of the energy landscape in Sec. III E.

\section{Migration of free oxygen vacancies}

As indicated in Fig. 1 there are two crystallographically distinct oxygen vacancy sites in the tetragonal perovskite lattice (space group P4mm). Vacancies can be situated along the $c$-axis $\left(V_{c}\right.$, Wyckoff site $\left.1 b\right)$ or within the $a b$-plane ( $V_{a b}$, Wyckoff site $\left.2 c\right) .{ }^{19,49,50}$ Thus, three different migration paths are possible between nearest neighbor sites: pure inplane migration $\left(V_{a b} \rightarrow V_{a b}\right)$, out-of-plane migration along the positive direction of the tetragonal axis $\left(V_{a b} \rightarrow V_{c}[001]\right)$, and out-of-plane migration along the negative direction of the tetragonal axis $\left(V_{a b} \rightarrow V_{c}[00 \overline{1}]\right)$.

The calculated barriers are compiled in Fig. 3(a). While in general migration barriers are charge state dependent, ${ }^{51,52}$ in the present case we have found the variations to be small. For migration within the $a b$-plane the barriers decrease as electrons are removed from the defect, which is in accord with the calculations on cubic perovskite structures. ${ }^{51,52}$ The barriers for migration via $c$-type vacancies, in contrast, increase. Since the charge state $q=+2$ prevails for the oxygen vacancy over the entire band gap the respective barriers were used in the construction of the energy landscapes serving as input for the kinetic model.

\section{Migration barriers for complexed oxygen vacancies}

The incorporation of an impurity on the $B$ site breaks translational symmetry along the tetragonal axis and lifts the degeneracy of the oxygen sites in this direction. One therefore obtains three distinct types of first-neighbor impurity atomoxygen vacancy associates (compare Figs. 1 and 3), which leads to three distinct migration paths. Migration within the second neighbor shell of impurity atoms was not considered, since it has been previously shown that the binding energy between oxygen vacancies and $\mathrm{Cu}$ and $\mathrm{Fe}$ impurities is the largest in the first neighbor shell. ${ }^{19}$ Thus, once an oxygen vacancy arrives in the vicinity of an impurity via diffusion, it will be attracted to the impurity and eventually reside in its first neighbor shell. The barriers for different migration paths of oxygen vacancies in the first nearest neighbor shell of copper and iron impurities are shown in Figs. 3(b)-3(d).

\section{E. Construction of energy landscape}

So far, we have calculated the migration barriers for free oxygen vacancies, which determine the elements of the rate matrix $\mathrm{K}_{i j}$ for $(i, j)=\{4,5\}$, and the migration barriers for oxygen vacancies in the first neighbor shell of an impurity atom, which provide the elements with $(i, j)=\{1,2,3\}$. Using these data some parts of the energy landscape can already be constructed as represented by the migration barriers shown in black in Fig. 4. 


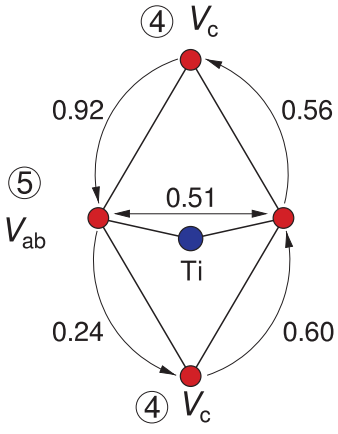

(a) free $V_{0}(q=+2)$

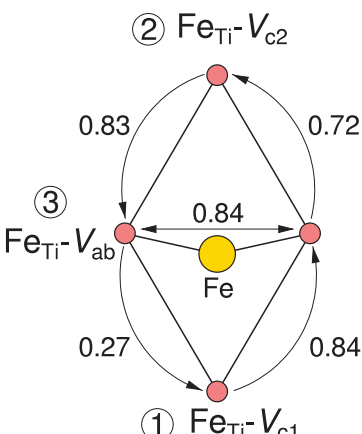

(c) $\mathrm{Fe}_{\mathrm{Ti}^{-}}-V_{\mathrm{O}}(\mathrm{q}=+1)$

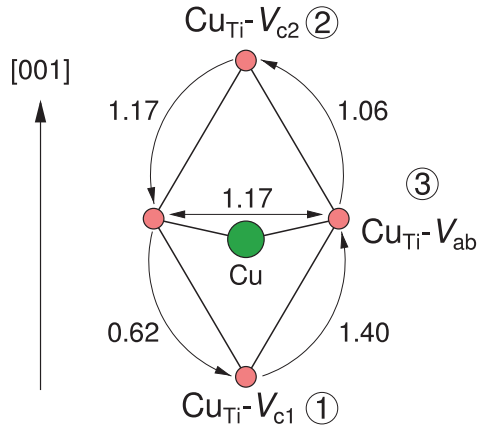

(b) $\mathrm{Cu}_{\mathrm{Ti}}-V_{\mathrm{O}}(\mathrm{q}=0)$

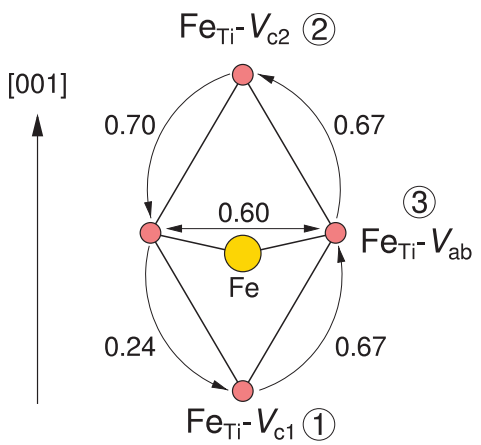

(d) $\mathrm{Fe}_{\mathrm{Ti}}-V_{\mathrm{O}}(\mathrm{q}=+2)$

FIG. 3. (Color online) Schematic representation of the barriers for the migration of free (a) and complexed (b)-(d) oxygen vacancies in units of $\mathrm{eV}$. Each figure shows the projection of a $\mathrm{BO}_{6}$ octahedron onto the (100) plane. The numbers in circles indicate the indices used to distinguish the different processes. Oxygen sites (and thus possible vacancy sites) are shown as red circles while the position of the $B$-site cation ( $\mathrm{Ti}, \mathrm{Cu}$, or $\mathrm{Fe}$ ), which is situated at the center of the oxygen octahedron, is indicated by blue (Ti), green $(\mathrm{Cu})$, and yellow $(\mathrm{Fe})$ circles.

The remaining combinations of $i$ and $j$, e.g., $1-5$ or $3-4$, correspond to transitions between free and complexed vacancies. In Sec. III B it was shown that the interaction between vacancy and impurity atom is short ranged as binding is very weak already for second and third nearest neighbor pairs. For the purpose of the present model we therefore assume that free vacancies transition via single hop into complexed vacancies.

To complete the rate matrix, we then assume the migration barriers for jumps between free oxygen vacancies to hold for jumps to complexed vacancies as well. The migration barriers inferred in this fashion are marked in green in Fig. 4. Note that the asymmetry found for the migration of free oxygen vacancies enters in so far as the barrier for a jump from $V_{a b}$ to $M_{B}-V_{c 1}\left(M_{B}-V_{c 2}\right)$ is given by the barrier for a free $a b$-type vacancy jumping with a component antiparallel (parallel) to the polarization direction.

To determine the barriers for the reverse jumps, we resort to the binding energies calculated previously for $\mathrm{Cu}_{\mathrm{Ti}}-V_{\mathrm{O}}$ (see Sec. III B) and $\mathrm{Fe}_{\mathrm{Ti}^{-}}-V_{\mathrm{O}}$ (see Ref. 19). These values are marked in blue in Fig. 4. For $\mathrm{Cu}$ and $\mathrm{Fe}$ the binding energies amount to $-1.35 \mathrm{eV}$ and $-1.00 \mathrm{eV}$, respectively, ${ }^{53}$ for Fermi levels near mid gap, which leads to the energy surfaces shown in Fig. 4. We have tested the sensitivity of the results of the kinetic

model to the barriers for jumps between free and complexed vacancies, which showed the assumptions made in determining their values to be of little consequence.

\section{RESULTS}

In this section we present results obtained using the kinetic model for $\mathrm{Cu}$ and Fe-doped lead titanate described in Sec. II. To illustrate the general features of vacancy redistribution, we first discuss the results for $\mathrm{Cu}$-doped $\mathrm{PbTiO}_{3}$ in the absence and presence of electric fields in Secs. IV A and IV B, respectively. The results for Fe-doped material are qualitatively very similar and will be summarized in Sec. IV C.

\section{A. Vacancy redistribution in the absence of electric fields}

Using the energy landscape for $\mathrm{Cu}$-doped $\mathrm{PbTiO}_{3}$ shown in Fig. 4 as input data for the kinetic model one can obtain the temporal evolution of the relative concentrations of different types of vacancies as exemplarily shown in Fig. 5(a) for a temperature of $300 \mathrm{~K}$ and a dopant concentration of $f_{M}=$ $5 \%$. In this example, the vacancies are initially statistically distributed over all available sites. In Fig. 5(a) four distinct time regimes with characteristically different dynamic balance can be identified that are separated by the transitions marked $\mathrm{A}, \mathrm{B}$, and $\mathrm{C}$ :

(i) The first regime (up to $t \lesssim 10^{-8} \mathrm{~s}$ at $300 \mathrm{~K}$ ) is associated with the redistribution of unbound vacancies. As can be seen from Fig. 4, $V_{c}$ is energetically preferred over $V_{a b}$. Figure 5(a) shows that even for a temperature as low as $300 \mathrm{~K}$ the redistribution between these two types of vacancies, i.e., the installation of the (partial) equilibrium over the subset of unbound vacancies, takes place within fractions of a second.

(ii) During the second stage $\left(10^{-8}-10^{-2} \mathrm{~s}\right.$ at $\left.300 \mathrm{~K}\right)$ unbound $c$-type vacancies dominate. Concurrently, dopants begin to capture vacancies. The dynamic equilibrium at this point is such that $\mathrm{Cu}_{\mathrm{Ti}}-V_{c 2}$ complexes dominate over $\mathrm{Cu}_{\mathrm{Ti}}-V_{c 1}$ and $\mathrm{Cu}_{\mathrm{Ti}}-V_{a b}$ dipoles. The former are energetically less favorable but are much more easily accessible since $\Delta E_{m}\left(V_{a b} \rightarrow\right.$ $\left.M_{B}-V_{c 2}\right)$ is only $0.24 \mathrm{eV}$ compared to $\Delta E_{m}\left(V_{a b} \rightarrow\right.$ $\left.M_{B}-V_{a b}\right)=0.51 \mathrm{eV}, \Delta E_{m}\left(V_{c} \rightarrow M_{B}-V_{a b}\right)=0.60 \mathrm{eV}$, and $\Delta E_{m}\left(V_{a b} \rightarrow M_{B}-V_{c 1}\right)=0.56 \mathrm{eV}$.

(iii) In the third stage $\left(10^{-2}-10^{7} \mathrm{~s}\right.$ at $\left.300 \mathrm{~K}\right)$ vacancy-dopant complexes take over with $\mathrm{Cu}_{\mathrm{Ti}^{-}}-V_{c 2}$ being the dominant defect. The prevalence of $\mathrm{Cu}_{\mathrm{Ti}}-V_{c 2}$ is inherited from the second stage which determines the initial concentrations for the third stage.

(iv) Eventually, the system reaches equilibrium $\left(t \gtrsim 10^{7} \mathrm{~s}\right.$ at $300 \mathrm{~K})$, i.e., virtually all vacancies occupy the lowest energy site. ${ }^{54}$

We can now discuss the dependence of vacancy migration on temperature and dopant concentration. As indicated by the letters A, B, and C in Fig. 5(a), characteristic times can readily be identified, at which the majority defect type changes. As shown in Fig. 5(b) the temperature dependence of these characteristic times can be fit to an Arrhenius equation, $\tau^{-1}=\omega_{0} \exp \left[-E_{A} / k_{B} T\right]$, using the migration barrier between the states involved as the activation energy. This analysis also demonstrates that the effect of changing the dopant concentration is small and is only visible for the transition between vacancy types $V_{c}$ and $\mathrm{Cu}_{\mathrm{Ti}}-V_{c 2}$. 


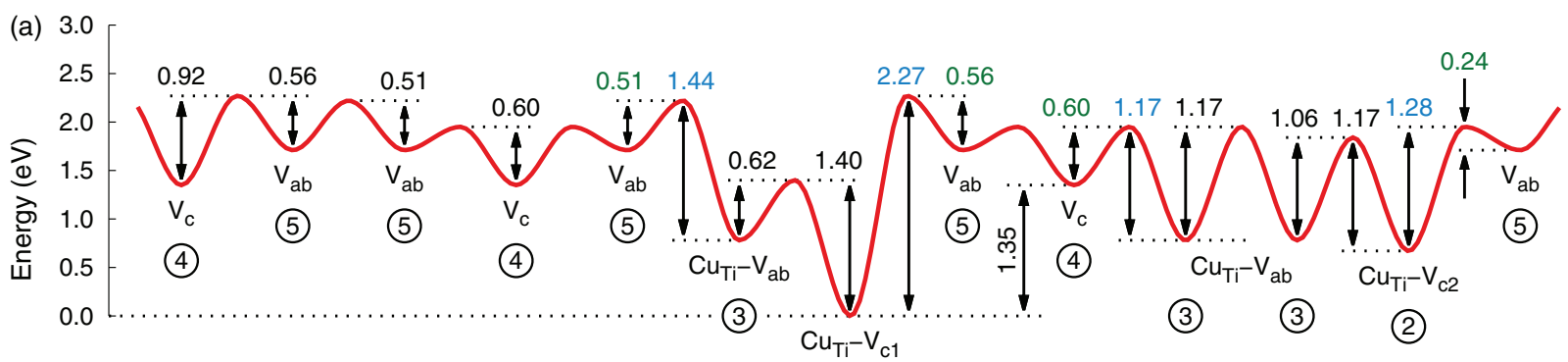

(1)

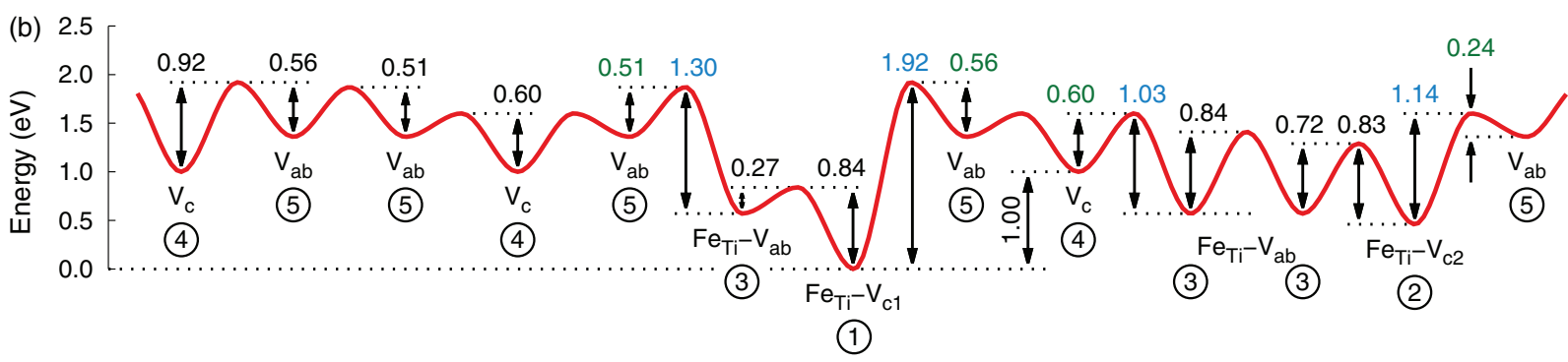

FIG. 4. (Color online) Energy surface for the migration of oxygen vacancies in (a) $\mathrm{Cu}$-doped $\left(\mathrm{Cu}_{\mathrm{Ti}}-V_{\mathrm{O}}, q=0\right)$ and (b) $\mathrm{Fe}$-doped $\mathrm{PbTiO}$ $\left(\mathrm{Fe}_{\mathrm{Ti}}-V_{\mathrm{O}}, q=+1\right)$. The sequence of barriers corresponds to a (hypothetical) continuous trajectory which illustrates all possible migration barriers (compare Figs. 1 and 3).

Already at temperatures $\gtrsim 450 \mathrm{~K}$ the full equilibrium is established within seconds. Since during growth these temperatures are easily reached, the vacancy distribution in tetragonal lead titanate should be in thermal equilibrium, i.e., virtually all dopants are complexed with vacancies in the ground state configuration, in which the defect dipoles are aligned with the domain polarization.

\section{B. Vacancy redistribution in the presence of electric fields}

In the present model the perturbation introduced by an external electric field enters in two ways. First the barriers for vacancy jumps with components along the direction of the electric field are distorted ("direct effect"), $\Delta E_{m} \rightarrow \Delta E_{m}-$ $\delta E$ where $\delta E=E_{\mathrm{loc}} \Delta r_{[001]} q e$. Here, $\Delta r_{[001]}$ denotes the displacement along the direction of the electric field which is positive (negative) if the displacement is parallel (antiparallel) to the electric field; $q$ is the charge state of the defect, $E_{\text {loc }}$ is the local electric field, and $e$ denotes the unit charge. Typical electric fields used for poling ferroelectric ceramics are on the order of $2-5 \mathrm{kV} / \mathrm{cm}$; the local electric field can, however, be larger than this value due to inhomogeneities. ${ }^{30}$ Assuming a value of $E_{\mathrm{loc}}=100 \mathrm{kV} / \mathrm{cm}$ and choosing $\Delta r_{[001]}=1 \AA$, we obtain an upper limit for $\delta E$ of $0.01 \mathrm{eV}$.

Obviously the "direct" effect of the electric field is rather small compared to the energy difference between different vacancy types and pertains to charged vacancies only. This implies that with regard to vacancy redistribution a material in a constant external field will behave almost identical to the situation without electric fields.

The situation does, however, change if we consider an oscillating external field. The field induced reversal of the polarization has a much larger effect on the energetics of the system than the direct contribution since reorientation of the polarization implies that the (average) displacement of $B$-site atoms is reversed, thus transforming $M_{B}-V_{c 1}$ into
$M_{B}-V_{c 2}$ complexes. In the present model, this is equivalent to exchanging rows 1 and 2 of the migration rate matrix $\mathrm{K}_{i j}$. One can therefore include the effect of an oscillating electric field by (i) periodically modifying the barriers for out-of-plane jumps by $\delta E$ and (ii) simultaneously exchanging the barriers for jumps involving $M_{B}-V_{c 1}$ or $M_{B}-V_{c 2}$.

Figure 6(a) illustrates the temporal evolution of the concentrations of $M_{B}-V_{c 1}$ and $M_{B}-V_{c 2}$ vacancies in the presence of an oscillating electric field. The plot shows that under prolonged cycling a dynamic balance between the two configurations $M_{B}-V_{c 1}$ and $M_{B}-V_{c 2}$ is established. As further illustrated in Fig. 6 the time after which this balance is obtained depends sensitively on temperature, e.g., at $340 \mathrm{~K}$ it is reached after approximately six days $\left(5 \times 10^{5} \mathrm{~s}\right)$ while at $380 \mathrm{~K}$ it requires only about two hours.

The dynamic balance between $M_{B}-V_{c 1}$ and $M_{B}-V_{c 2}$ occurs because the characteristic time required to reach full equilibrium in the absence of external electric fields [see Fig. 5(b)] exceeds the cycling period. In a fast switching field the restoring force of the spontaneous polarization is changing signs on a short time scale. As a result, a mean field composed of parallel and antiparallel polarization of the matrix is acting on the dipoles, which eventually populate both orientations along the $c$ axis. This dynamic equilibrium is sensitive to temperature and the assumption of a static distribution of defect dipoles in a fast switching field should be taken with care. The results show that by applying a bipolar electric field defect dipoles are redistributed and on average the clamping effect on domain walls is reduced. This is in line with recent results on deaging of doped $\mathrm{PZT} .^{29,30}$

\section{Results for Fe-doped lead titanate}

Complexes of Fe with oxygen vacancies act as donors leading to electron chemical potentials in the upper half of the band gap. The most stable charge state is $q=+1 .^{19}$ 

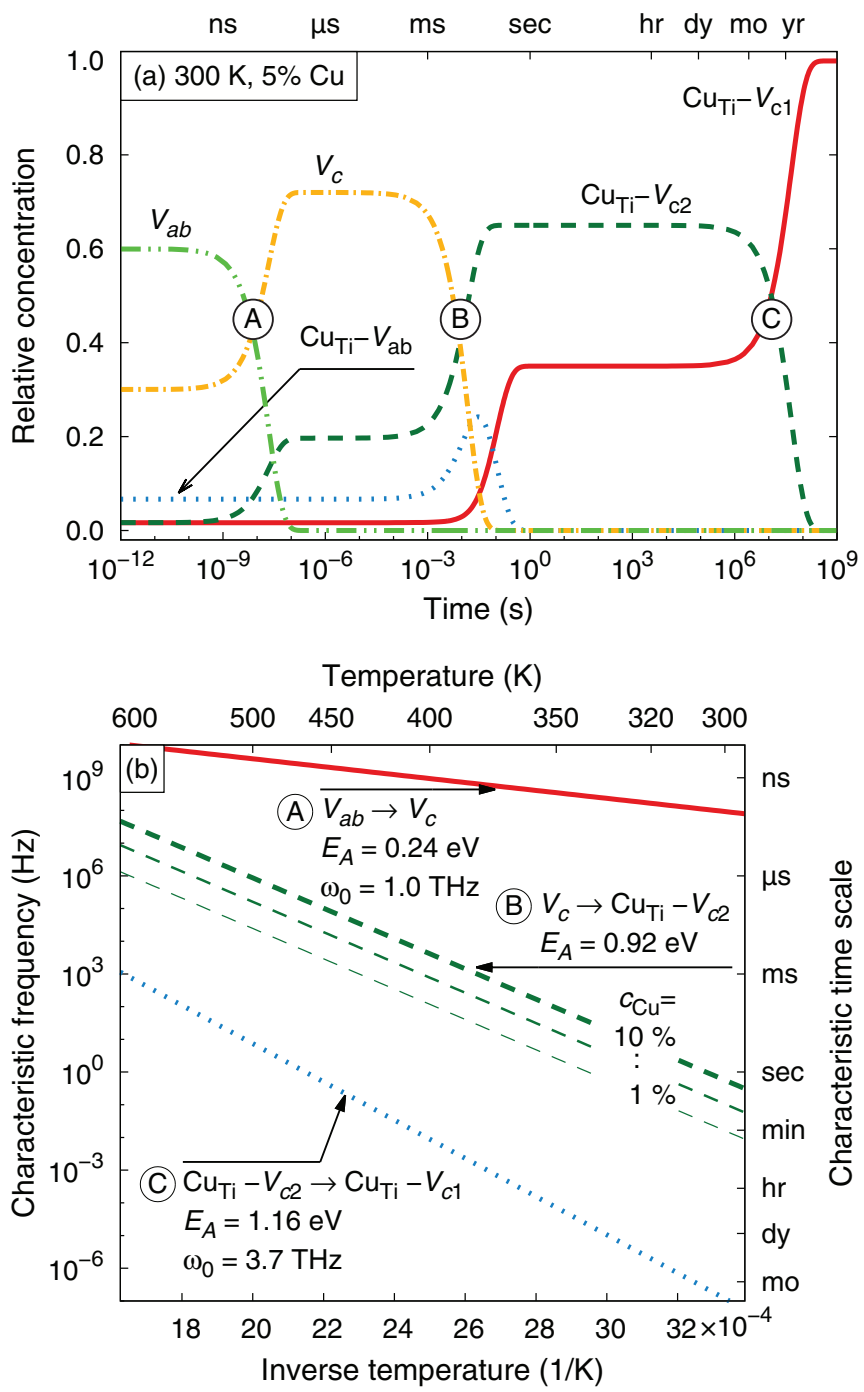

FIG. 5. (Color online) Vacancy redistribution in the absence of electric fields for $\mathrm{Cu}$-doped lead titanate: (a) Temporal evolution of the relative concentrations of different vacancy types at a temperature $300 \mathrm{~K}$ and for a $\mathrm{Cu}$ concentration of $5 \%$. (b) Temperature dependence of the characteristic transition times marked by white circles in (a). The transition time for the conversion from $V_{c}$ to $\mathrm{Cu}_{\mathrm{Ti}}-V_{c 2}$ depends on the $\mathrm{Cu}$ concentration as exemplified by the three green dashed lines of varying thickness.

The energy landscape for these conditions is shown in Fig. 4(b).

The temporal evolution of different vacancy configurations in the absence of an external electric field is shown in Fig. 7(a) which allows us to infer the temperature dependence of the characteristic time scales summarized in Fig. 7(b). Comparing Figs. 5 and 7 we find that the results for $\mathrm{Fe}$ - and $\mathrm{Cu}$-doped lead titanate are very similar. This is expected since the first two transitions $\left(V_{a b} \rightarrow V_{c}\right.$ and $V_{c} \rightarrow \mathrm{Fe}_{\mathrm{Ti}}-V_{c 2}$, compare Sec. IIIE) are determined by the migration barriers in the pure host. With regard to the third transition between $M_{B}-V_{c 2}$ and $M_{B}-V_{c 1}$ the situation is different as the effective barrier in Fedoped material is $0.83 \mathrm{eV}$ and thus smaller than in $\mathrm{Cu}$-doped lead titanate [1.16 eV, compare Figs. 3 and 5(b)], which speeds up the transition. We can thus expect that in the presence of
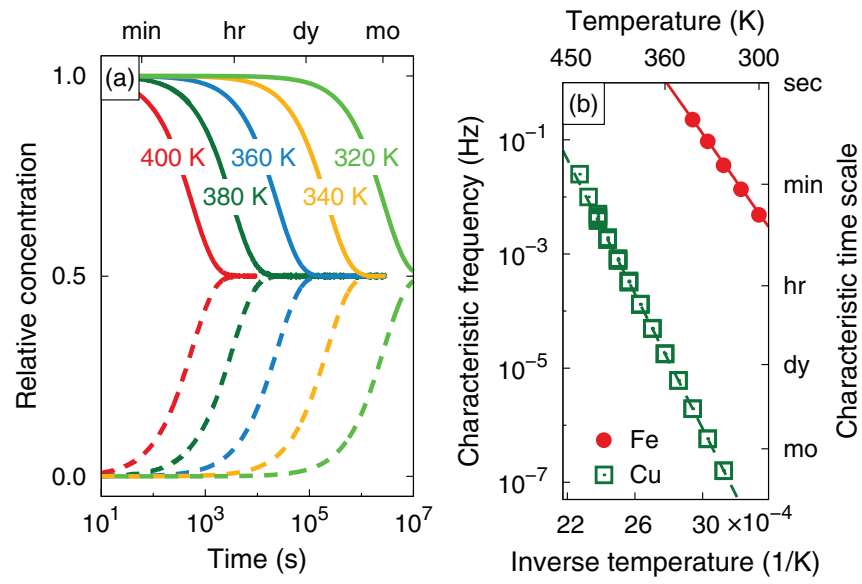

FIG. 6. (Color online) Vacancy redistribution in the presence of an oscillating external field: (a) Equilibration over vacancy types $\mathrm{Cu}_{\mathrm{Ti}}-V_{c 1}$ (solid lines) and $\mathrm{Cu}_{\mathrm{Ti}}-V_{c 2}$ (dashed lines) in the presence of an external oscillating field with a cycling frequency of $1 \mathrm{~Hz}$ for temperatures between 320 and $400 \mathrm{~K}$. (b) Temperature dependence of time after which the relative concentration of $M_{B}-V_{c 1}$ complexes is reduced to $55 \%$ where $M$ is either $\mathrm{Cu}$ or $\mathrm{Fe}$.

an oscillating external field the dynamic equilibrium between $\mathrm{Fe}_{\mathrm{Ti}}-V_{c 2}$ and $\mathrm{Fe}_{\mathrm{Ti}}-V_{c 1}$ is established faster as well, which is confirmed by explicit calculation [see Fig. 6(b)]. Whereas for $\mathrm{Cu}$-doped lead titanate our model calculations predict the equilibrium near room temperature to be installed over a period of weeks and months, in Fe-doped material the same process should occur on the order of minutes.

\section{DISCUSSION}

We have parametrized a kinetic model for defect dipole formation and switching by taking data from first-principles calculations for $\mathrm{Fe}$ - and $\mathrm{Cu}$-doped $\mathrm{PbTiO}_{3}$. We find that at temperatures $\gtrsim 450 \mathrm{~K}$, which is well below the Curie temperature of $720 \mathrm{~K},{ }^{55}$ the formation and alignment of defect dipoles in doped $\mathrm{PbTiO}_{3}$ should occur within less than a second. ${ }^{56}$

Bipolar poling leads to a dynamic equilibrium between defect dipoles that are aligned parallel and antiparallel to the lattice polarization, respectively, and thus can be seen as one major contribution to deaging of PZT ceramics. This is in accord with the experimental observation of deaging by the application of $\mathrm{AC}$ fields. ${ }^{29,30}$ In $\mathrm{Cu}$-doped lead titanate the dynamic equilibration takes about two weeks at $300 \mathrm{~K}$, but is massively accelerated if temperature is slightly increased. This points to the importance of closely monitoring the sample temperature during testing and studying aging and deaging processes.

For the $M_{B}-V_{c 1}$ complex, which for both $\mathrm{Cu}$ and $\mathrm{Fe}$ is the ground state configuration, the local polarization is parallel to the polarization of the surrounding matrix. ${ }^{19}$ Since in contrast $M_{B}-V_{c 2}$ defects are aligned antiparallel to the lattice polarization, an increase in their concentration causes an overall loss of switchable polarization. This direct contribution should scale linearly with the number of impurity atoms in the sample but due to the small magnitude of the defect dipole moment will amount to a rather small contribution on the 

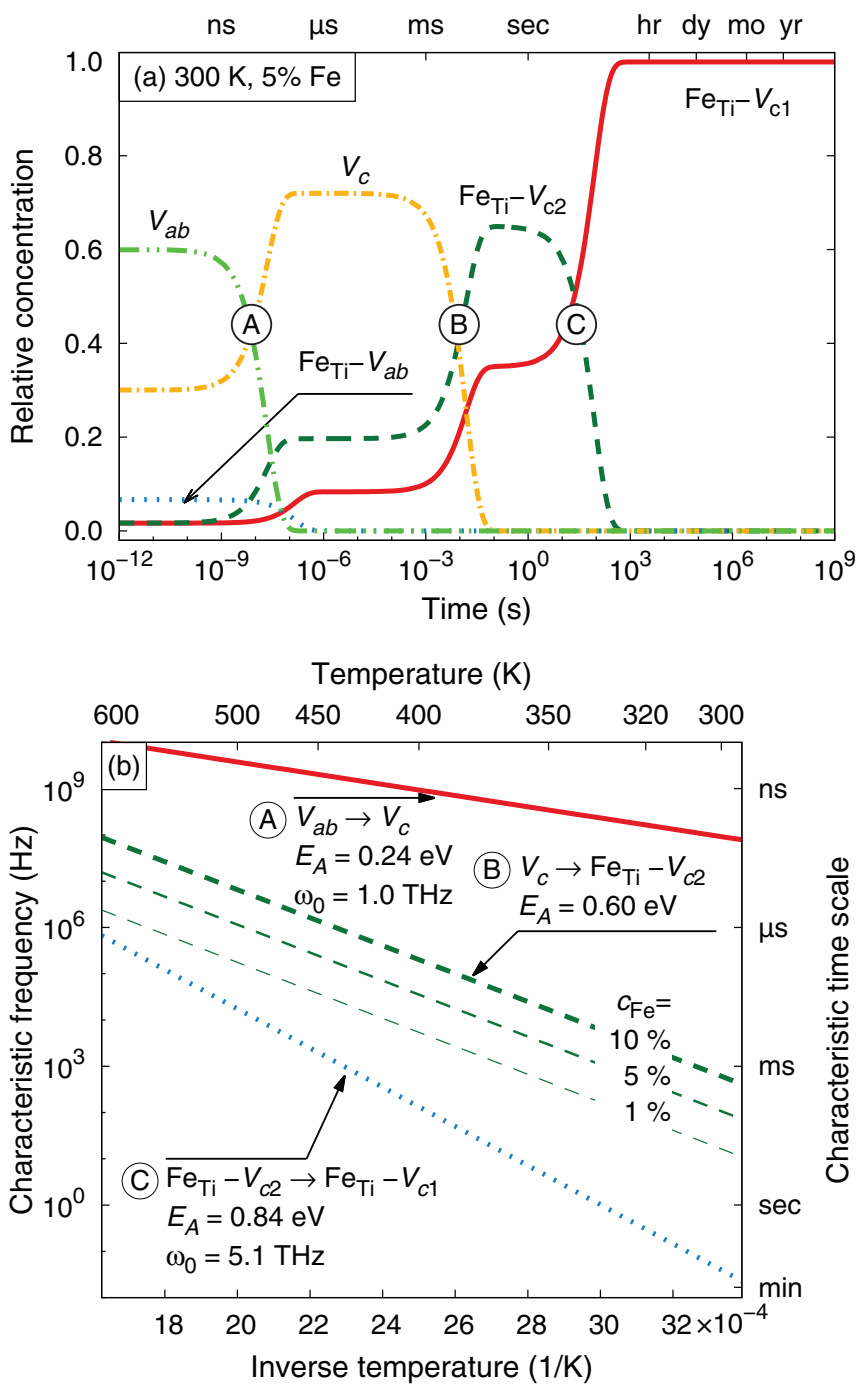

FIG. 7. (Color online) (a) Temporal evolution of the relative concentrations of different vacancy types for three different temperatures in Fe-doped lead titanate. (b) Dependence of characteristic time scales for the transitions indicated by white circles in (a) on temperature and dopant concentration.

macroscopic scale. Defect dipoles, however, also interact with domain walls and can affect their mobility. In lead titanate and tetragonal PZT one typically observes $90^{\circ}$ domain wall configurations, which is schematically indicated in Fig. 8. It has been shown by first-principles calculations that the head-to-tail domain wall configuration shown, e.g., in Fig. 8, is energetically more stable than head-to-head or tail-to-tail configurations. ${ }^{57}$ In the pristine material after cooling (middle panel of Fig. 8) all defect dipoles are aligned with the lattice polarization and thus follow the head-to-tail pattern. This situation changes significantly after cyclic loading (right panel of Fig. 8) since now half of the defect dipoles oppose the lattice polarization and thus create local high-energy head-to-head and tail-to-tail configurations.

Recent simulations of domain wall motion using an empirical force field ${ }^{16}$ have provided impressive evidence that domain wall motion proceeds via a nucleation-and-growth process. It will be the subject of future work to determine the
Initial state at high T

dipole orientation random

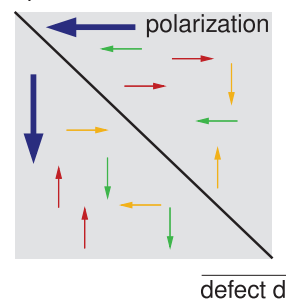

After cooling

dipoles aligned with $P_{s}$

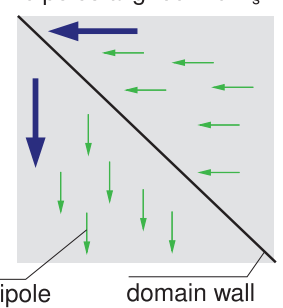

After cyclic loading

50-50 alignment

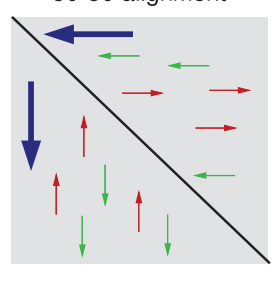

FIG. 8. (Color online) Schematic representation of defect dipole arrangements under different conditions as deduced from the kinetic model. The large value arrows indicate the matrix polarization in different domains while the small arrows represent defect dipoles. The thick solid line illustrates the position of a $90^{\circ}$ domain wall.

role of defect dipoles quantitatively. At the present stage one can already imagine that defect dipoles aligned antiparallel to the local lattice polarization will impede both nucleation and growth of domains while the opposite can be said for defect dipoles that are aligned parallel to the lattice polarization. Even though the direct contribution of defect dipoles to the macroscopic polarization is small they can have a significant indirect impact by pinning domain walls and reducing their mobility. The fact that domain motion occurs via nucleation and growth is crucial in this context since it implies that domain wall motion occurs locally and can thus be strongly influenced by localized defect dipoles.

\section{CONCLUSIONS}

In the present work we have derived a kinetic model that allows us to study the temporal evolution of defect dipoles in the absence and presence of electric fields. The most important input parameter is the energy landscape for oxygen vacancy migration. Using parameters for $\mathrm{Cu}$ - and $\mathrm{Fe}$-doped $\mathrm{PbTiO}_{3}$ obtained from density functional theory calculations, we found that the equilibration of the vacancy distribution occurs readily at temperatures considerably below the Curie temperature. As a result in the as-synthesized material virtually all impurity atoms are associated with vacancies forming $M_{B}-V_{c 1}$ complexes. The complete realignment of vacancymetal impurity dipoles parallel to the spontaneous polarization occurs on time scales of hours to days at room temperature, but is massively accelerated if temperature is slightly increased. This provides evidence for the fact that aging due to defect dipoles occurs instantaneously in $\mathrm{PbTiO}_{3}$-based ferroelectrics.

In the presence of an oscillating electric field a dynamic balance between $M_{B}-V_{c 1}$ and $M_{B}-V_{c 2}$ is established. Prolonged cycling therefore leads to the accumulation of defect dipoles that oppose the polarization of the encompassing domain. While these defect dipoles directly reduce the switchable polarization, more importantly they can impede domain wall motion, which has been recently shown to proceed via nucleation and growth. ${ }^{16}$

The present results provide valuable insights into the switching kinetics of defect dipoles in ferroelectrics, which is relevant for understanding aging and deaging mechanisms. 
The rate equation approach can be adapted straightforwardly to describe more complex geometries and systems. This could be used to model the lattice geometries of, e.g., $\mathrm{BiFeO}_{3}$ or $\mathrm{LaMnO}_{3}$.

Kinetic models similar to the one discussed in the present paper can also be used to interpret experimental measurements. To this end, one could employ probes which are sensitive to the orientation of the defect dipoles (e.g., electron spin resonance $^{18,23}$ ) and measure the intensity of the signal before, during, and after cycling or heat treatments.

\section{ACKNOWLEDGMENTS}

This project was partially funded by the Sonderforschungsbereich 595 "Fatigue in functional materials" of the Deutsche Forschungsgemeinschaft. P.E. acknowledges funding from the "Areas of Advance-Materials Science" at Chalmers and the European Research Council in the Form of a Marie Curie Career Integration Grant. Computer time allocations by the Swedish National Infrastructure for Computing at C3SE (Gothenburg) and PDC (Stockholm) are gratefully acknowledged. *erhart@chalmers.se

${ }^{1}$ K. W. Plessner, Proc. Phys. Soc. London, Sect. B 69, 1261 (1956).

${ }^{2}$ S. Ikegami and I. Ueda, J. Phys. Soc. Jpn. 22, 725 (1967).

${ }^{3}$ K. Carl and K. Härdtl, Ferroelectrics 17, 473 (1978).

${ }^{4}$ S. Takahashi, Ferroelectrics 41, 143 (1982).

${ }^{5}$ G. Arlt and H. Neumann, Ferroelectrics 87, 109 (1988).

${ }^{6}$ R. Lohkamper, H. Neumann, and G. Arlt, J. Appl. Phys. 68, 4220 (1990).

${ }^{7}$ W. L. Warren, D. Dimos, G. E. Pike, K. Vanheusden, and R. Ramesh, Appl. Phys. Lett. 67, 1689 (1995).

${ }^{8}$ V. P. Afanasjev, A. A. Petrov, I. P. Pronin, E. A. Tarakanov, E. J. Kaptelov, and J. Graul, J. Phys.: Condens. Matter 13, 8755 (2001).

${ }^{9}$ L. X. Zhang and X. Ren, Phys. Rev. B 71, 174108 (2005).

${ }^{10}$ L. Zhang, E. Erdem, X. Ren, and R.-A. Eichel, Appl. Phys. Lett. 93, 202901 (2008).

${ }^{11}$ M. I. Morozov and D. Damjanovic, J. Appl. Phys. 104, 034107 (2008).

${ }^{12}$ Y. A. Genenko, J. Glaum, O. Hirsch, H. Kungl, M. J. Hoffmann, and T. Granzow, Phys. Rev. B 80, 224109 (2009).

${ }^{13}$ L. Zhang and X. Ren, Func. Mater. Lett. 3, 69 (2010).

${ }^{14}$ A. Tagantsev, I. Stolichnov, E. Colla, and N. Setter, J. Appl. Phys. 90, 1387 (2001).

${ }^{15}$ X. Ren, Nat. Mater. 3, 91 (2004).

${ }^{16}$ Y.-H. Shin, I. Grinberg, I.-W. Chen, and A. M. Rappe, Nature (London) 449, 881 (2007).

${ }^{17}$ C.-L. Jia, S.-B. Mi, K. Urban, I. Vrejoiu, M. Alexe, and D. Hesse, Nat. Mater. 7, 57 (2008).

${ }^{18}$ H. Meštrić, R.-A. Eichel, T. Kloss, K.-P. Dinse, S. Laubach, S. Laubach, P. C. Schmidt, K. A. Schönau, M. Knapp, and H. Ehrenberg, Phys. Rev. B 71, 134109 (2005).

${ }^{19}$ P. Erhart, R.-A. Eichel, P. Träskelin, and K. Albe, Phys. Rev. B 76, 174116 (2007).

${ }^{20}$ S. Pöykkö and D. J. Chadi, Phys. Rev. Lett. 83, 1231 (1999).

${ }^{21}$ H. Meštrić, R.-A. Eichel, K.-P. Dinse, A. Ozarowski, J. van Tol, and L. Brunel, J. Appl. Phys. 96, 7440 (2004).

${ }^{22}$ A. Boonchun, M. F. Smith, B. Cherdhirunkorn, and S. Limpijumnong, J. Appl. Phys. 101, 043521 (2007).

${ }^{23}$ Rudiger-A. Eichel, P. Erhart, P. Träskelin, K. Albe, H. Kungl, and M. J. Hoffmann, Phys. Rev. Lett. 100, 095504 (2008).

${ }^{24}$ P. Marton and C. Elsässer, Phys. Rev. B 83, 020106 (2011).

${ }^{25}$ H. Neumann and G. Arlt, Ferroelectrics 76, 303 (1987).

${ }^{26}$ In Ref. 5 an alternative estimate for the energy differences between different defect dipole alignments is given based on dipolar interaction, which leads to larger energy differences that are closer to the ones obtained by first-principles calculations. These values were, however, not employed in said reference to actually model aging.

${ }^{27}$ P. Jakes, E. Erdem, R.-A. Eichel, L. Jin, and D. Damjanovic, Appl. Phys. Lett. 98, 072907 (2011).

${ }^{28}$ Since the direct contribution of the electric field to the energy landscape is very small (see Sec. IV B), within our model the results for nonoscillating (DC) fields are virtually identical to the situation without any external field.

${ }^{29}$ T. Granzow, E. Suvaci, H. Kungl, and M. J. Hoffmann, Appl. Phys. Lett. 89, 262908 (2006).

${ }^{30}$ J. Glaum, Y. A. Genenko, H. Kungl, L. A. Schmitt, and T. Granzow, J. Appl. Phys. 112, 034103 (2012).

${ }^{31}$ In principle, the solution of Eq. (5) can be written in terms of the eigenvalues and vectors of $\mathbf{W}^{\mathrm{T}}-\mathbf{V}$. Since the eigenvalues appear in an exponential function, the stability of the solution, which can be tested via Eq. (2), is highly sensitive to their numerical accuracy. In practice, we have therefore resorted to numerical solvers that approach Eq. (5) directly.

${ }^{32}$ Specifically, we used the ode15s solver of MATLAB (Ref. 58).

${ }^{33}$ G. H. Vineyard, J. Phys. Chem. Solids 3, 121 (1957).

${ }^{34} \mathrm{G}$. Wahnström, Interactions of Atoms and Molecules with Solid Surfaces (Plenum Press, New York, 1990), p. 529.

${ }^{35}$ P. G. Sundell, M. E. Björketun, and G. Wahnström, Phys. Rev. B 76, 094301 (2007).

${ }^{36}$ P. Ghosez, E. Cockayne, U. V. Waghmare, and K. M. Rabe, Phys. Rev. B 60, 836 (1999).

${ }^{37}$ G. Kresse and J. Hafner, Phys. Rev. B 47, 558 (1993); 49, 14251 (1994); G. Kresse and J. Furthmüller, ibid. 54, 11169 (1996); Comput. Mater. Sci. 6, 15 (1996).

${ }^{38}$ P. E. Blöchl, Phys. Rev. B 50, 17953 (1994); G. Kresse and D. Joubert, ibid. 59, 1758 (1999).

${ }^{39}$ D. M. Ceperley and B. J. Alder, Phys. Rev. Lett. 45, 566 (1980); J. P. Perdew and A. Zunger, Phys. Rev. B 23, 5048 (1981).

${ }^{40}$ A. M. Glazer and S. A. Mabud, Acta Crystallogr. B 34, 1065 (1978).

${ }^{41}$ J. Robertson and C. W. Chen, Appl. Phys. Lett. 74, 1168 (1999).

${ }^{42}$ P. Erhart and K. Albe, Phys. Rev. B 73, 115207 (2006).

${ }^{43}$ G. Henkelman, G. Jóhannesson, and H. Jónsson, in Progress on Theoretical Chemistry and Physics (Kluwer Academic, Dordrecht, 2000), p. 269.

${ }^{44}$ G. Henkelman, B. P. Uberuaga, and H. Jónsson, J. Chem. Phys. 113, 9901 (2000).

${ }^{45}$ S. Gottschalk, H. Hahn, S. Flege, and A. G. Balogh, J. Appl. Phys. 104, 114106 (2008).

${ }^{46}$ The initial distance vectors in the second neighbor configurations that are unstable are $\left\langle 0 \frac{1}{2} 1\right\rangle,\left\langle 0 \frac{1}{2} \overline{1}\right\rangle$, and $\left\langle 1 \frac{1}{2} 0\right\rangle$. 
${ }^{47}$ The initial distance vectors in the second neighbor configurations that are stable are $\left\langle 10 \frac{1}{2}\right\rangle$ and $\left\langle 10 \frac{\overline{1}}{2}\right\rangle$.

${ }^{48}$ Note that in Ref. 19 we obtained a binding energy of $2.3 \mathrm{eV}$. The large difference to the present value is due to an unfortunate addition of errors. In Ref. 19 the error due to image charge interactions was estimated using the monopole-monopole term of the Makov-Payne expansion (Ref. 59), which is known to overestimate the interaction and gives a value of $\Delta E_{\text {corr }}=0.11 \mathrm{eV}$. The binding energy of $\mathrm{Cu}_{\mathrm{Ti}}-V_{\mathrm{O}}$ was obtained as the difference between the formation energies of a neutral complex and two doubly charge isolated defects, which effectively lead to the inclusion of a "correction" term of $2 \times 2^{2} \Delta E_{\text {corr }} \approx 0.9 \mathrm{eV}$ in the binding energy. Using the larger supercells available in the present study we are able to calculate the binding energy directly (see Fig. 2) and revisit the previous calculation as well as the error estimate. This shows the earlier "correction" to be almost exclusively responsible for the difference between the binding energies computed in Ref. 19 and here. Since in either case binding is very strong both compared to $k_{B} T$ and the migration barriers, none of the conclusions in Ref. 19 are affected. Also note that the binding energies for $\mathrm{Fe}_{\mathrm{Ti}}-V_{\mathrm{O}}$ complexes are not affected.

${ }^{49}$ C. H. Park and D. J. Chadi, Phys. Rev. B 57, R13961 (1998).

${ }^{50}$ Park and $\mathrm{Chady}^{49}$ discuss two different configurations for the $a b$ site vacancy, $V_{a b}^{s w}$ and $V_{a b}^{u d}$, but the former one seems to be always lower in energy and therefore prevails. We are thus left with only two different types vacancies, $V_{c}$ and $V_{a b}$. Also compare the discussion in Ref. 19.
${ }^{51}$ C. H. Park, J. Korean Phys. Soc. 42, S1420 (2003).

${ }^{52}$ P. Erhart and K. Albe, J. Appl. Phys. 102, 084111 (2007).

${ }^{53} \mathrm{In}$ the case of $\mathrm{Fe}$ the binding energy varies with the electron chemical potential but remains negative within the band gap. The value of $-1.00 \mathrm{eV}$ used here is representative. The results obtained in Sec. IV are insensitive to this choice as long as the three nearest neighbor complexes are energetically lower than $V_{c}$ [compare Fig. 4(b)]. This condition is fulfilled for electron chemical potentials above approximately $0.3 \mathrm{eV}$ (measured with respect to the valence band edge), which should be the case anyway since Fe-vacancy complexes act as donors and thus rather push the Fermi level to the conduction band side.

${ }^{54}$ At finite temperatures a fraction of vacancies will also occupy higher energy configurations. Their number, however, is very small and indistinguishable from zero on the scale of Fig. 5(a), since it is determined by the Boltzmann factor $\exp \left(-\Delta E / k_{B} T\right)$ where $\Delta E$ is the energy difference between the ground state configuration, $M_{B}-V_{c 1}$, and the configuration in question.

${ }^{55}$ B. Noheda, N. Cereceda, T. Iglesias, G. Lifante, J. A. Gonzalo, H. T. Chen, and Y. L. Wang, Phys. Rev. B 51, 16388 (1995).

${ }^{56} \mathrm{The}$ Curie temperatures of $\mathrm{PbTiO}_{3}$ and $\mathrm{PbZrO}_{3}$ are 720 and $460 \mathrm{~K}$, respectively (Ref. 55), and the concentration dependent Curie temperature of PZT is bounded by these values.

${ }^{57}$ B. Meyer and D. Vanderbilt, Phys. Rev. B 65, 104111 (2002).

${ }^{58}$ MATLAB, version 7.10.0 (R2010a) (The MathWorks Inc., Natick, Massachusetts, 2010).

${ }^{59}$ G. Makov and M. C. Payne, Phys. Rev. B 51, 4014 (1995). 